\title{
Innovative Metaphorical Design based on Parametric Technology
}

\author{
Tian Jiale ${ }^{1 s t, a}$, Zeng Li ${ }^{2 n d, b}$ \\ ${ }^{1}$ School of Furniture and Art Design, Central South University of Forestry Science and Technology, Changsha, Hunan, China \\ ${ }^{2}$ School of Furniture and Art Design, Central South University of Forestry Science and Technology, Changsha, Hunan, China
}

\begin{abstract}
This paper explores the application of metaphorical design in furniture design based on parametric technology. The semantics of designs were expressed by metaphors to enrich the ways of expression of furniture and meet customized needs. The methods, application and emotional expression of metaphorical design were analyzed, and the utilization of parametric technology in metaphorical design was explored based on case studies. Metaphorical design, as a means of furniture design, is a way of expression, or even an outcome of expression. Use of metaphorical design in the design process and parametric technology in manufacturing will allow the designers to turn conceptual ideas into real furniture pieces.
\end{abstract}

\section{INTRODUCTION}

Despite the rapid advancements in technology, the modern society is facing increasing mental pressure and the younger generations prefer staying alone instead of going out to socialize. This preference has also found its presence in design of products. With more time spent at home, people need more emotional accompanying, looking for something "intangible" that can be emotions or technologies. Then, we have to consider how to facilitate communication between the products and the consumers, how to align the products with the consumers' emotional needs, and how to encourage the consumers to embrace new technology. Metaphorical design provides a solution to these challenges.

\section{CONCEPT AND CONNOTATION OF METAPHORICAL DESIGN}

Metaphor refers to the conversion of meaning. Metaphor is a rhetorical device that replaces one image with another, with the essential meaning unchanged ${ }^{[1]}$.Metaphor is a means to help people understand and convey emotions. It is not only used in literature, but also widely used in design, with high practical value ${ }^{[2]}$. Metaphorical design in furniture design is to use the method of metaphor to process the elements of furniture products, which does not change the essential meaning of the products, thus to fulfill people's emotional needs.

The purpose of metaphorical design is to speed up cognition and convey emotions. Cognition is driven by the users' needs and interests. In metaphorical design, it is necessary to capture the users' interests and emotional needs, thereby encouraging them to understand the design and promoting technological development.

\section{MetAPHORICAL DESIGN OF FURNITURE}

\subsection{Methods of furniture metaphorical design}

The external form of the product itself serves cognitive and communicative functions. Furniture, as a form of articles for daily use, serves basic functions and interacts with the users in application ${ }^{[3]}$. Furniture articles come in different shapes, showing different spirit and strength. Physical characteristics, color schemes and raw materials are all factors that affect the metaphorical effect of the shape of furniture. In metaphorical design of furniture, elements familiar to users should be employed to reduce the learning and cognitive pressure of new products to give the users a feeling that everything is under their control. Metaphorical design of the shape of furniture is an effective way to generate rich connotations. The metaphorical design methods are as follows ${ }^{[4]}$.

By collecting or producing relevant metaphors, the designer determines the metaphorical source by analyzing the user's emotion or his inner world, and projects the emotion, culture and spirit onto the product, so that the form of the product can deliver a message and in-depth emotions to increase its appeal to the customers. As the saying goes, there are a thousand Hamlets in a thousand people's eyes. The success of a metaphorical design depends on whether it can stir up imaginations among the users.

In metaphorical design, the designer takes advantage of the similarity between different objects, and highlight the salient features (physical characteristics, colors, cognition or materials) of the metaphorical object to reflect on the shape of the product. What is the definition of "similarity"? Is there a range? The correlation between a concept and its extension consists of a range. Take the design of a chair for example. A chair does not necessarily

ae-mail: 935396550@qq.com

b*Corresponding author: e-mail jenny6663@163.com 
have a backrest, and any object that can serve the function of "sitting" can be called a chair, like a glider or a recliner, because they share the same internal attribute. The range can be defined by similarity.

\subsection{Expression of metaphorical design of furniture}

\subsubsection{Emotional metaphors for physical characteristics}

\subsubsection{The emotional metaphor of modeling}

Line: There are different types of lines, like straight lines, curves, or a mix of curves and lines. In design, lines can be used for partitioning or decoration. The length, thickness and state of the line will create different psychological feelings in the users. Straight lines are simple and bright. For example, design of chairs in Ming and Qing Dynasties shows extensive use of lines and a 90degree backrest. In ancient China, Confucianists and Taoists seek to expose the inner spirit, so design of products often aims to demonstrate the quality, temperament and generosity of the user. Such design philosophy has been introduced to design of furniture and thus the furniture articles emit a sense of calm and integrity. Compared with straight lines, curves are softer, more elegant, and full of changes, embodying the female plumpness and the flexible ease of flowing water.

Surface: The outer surface of the design interacts with users, and from it, the users knows what the product is used for, what functions it serves and whether it is safe. For instance, round and square tables are more suitable to serve as dining tables than tables with irregular shapes, and their design ideas derive from the idioms like "the sky is round and the earth is square" and "all is smooth". Round tables symbolize completeness and square tables symbolize safety. Irregularly-shaped tables give a feeling of threats and are likely to cause disputes in the family.

Body: Metaphorical design creates a physical "resemblance". Some bionic and nature-like concrete designs extract elements from the nature. The nature offers many inspirations. For instance, the Luban saw was inspired by the shape of grass veins; the Peacock Chair resembles the spreading of the tail of a peacock. This chair, designed by a Canadian company called Uufie, is an imitation of an animal. The shape of the chair resembles a peacock showing its tails, flaunting and gorgeous, posing a strong feeling of the queen, as if saying "I am noble".

\subsubsection{Emotional metaphor of raw materials}

Materials have their own visual and tactile characteristics, and different materials can cause different feelings. For instance, wood gives a person a kind of natural feeling, fabric gives a feeling of softness, metals have an industrial feeling, and bamboo rattan gives a rustic feeling. Raw materials for furniture design have been extended as the time moves forward, and eco-friendly materials are reaching maturity. What can we do to alleviate the problem of global warming in design? Metaphorical designs with eco-friendly materials reflect people's sense of environmental protection and their resolve to address the problem of depletion of resources on earth. Bentu Design, for example, uses waste concrete and ceramic tiles and recycled building wastes in their designs, and designs chairs that fit the people's needs by taking advantage of the strong plasticity of the concrete. (Figs. 1,2)

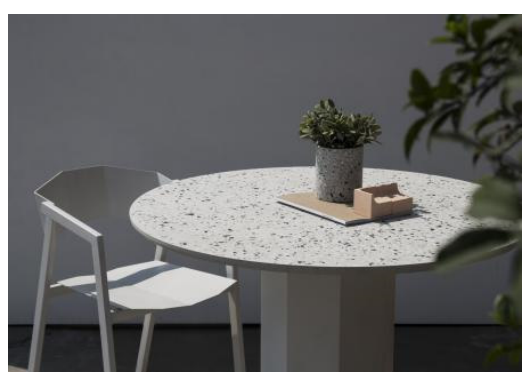

Fig. 1 Bentu Design works

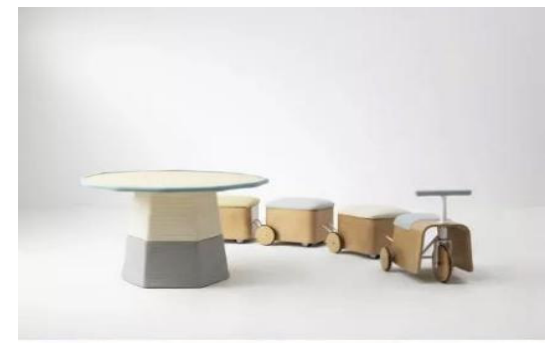

Fig. 2 The chair

\subsubsection{Emotional metaphor of colors}

Living in a colorful world, we are used to being surrounded by colors, but colors also can also be used as metaphors. Colors causes visual effects that impact the human brain. Yet, colors do not exist alone, and when attached to products, they cause different emotions. Colors are linked with emotions. Just like the color schemes on the food packages, the colors of the furniture also evoke different sensory and visual effects. Colors can also bring about different psychological effects as the age, the emotion and the character of the user varies. Thus, a piece of furniture is often a blend of different colors to give a feeling of vigor and encourage the customers to buy. To reach this end, the designer needs to have keen insights into design.

\subsubsection{Emotional metaphor of psychological characteristics}

Metaphor produces a psychological "resemblance", which can convey emotions and map the original cognition onto the product. Human creation presupposes imitation. Furniture designers, by imitating the colors and shapes from the nature, applies the inspirations to life. For example, the womb chair designed by Eero Saari - nen in 1946 (Fig. 4) uses cashmere as the raw material and combines nature with furniture. It gives a feeling of warmth and comfort. When sitting on it, the user is like 
being embraced by his/her mom, warm and caring. The chair resonates with the user's needs and gives a feeling of safety at home.

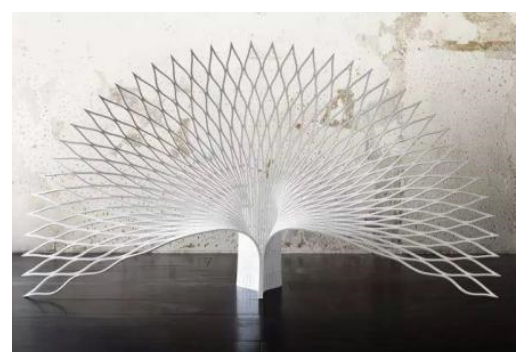

Fig. 3 Angled four-lobed window

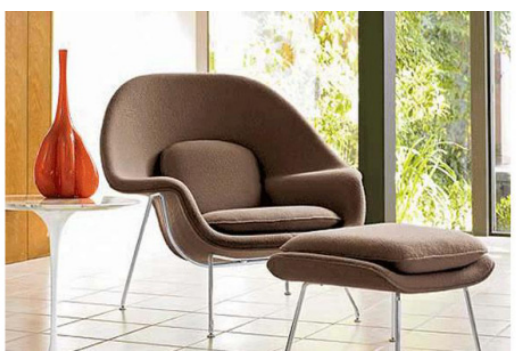

Fig. 4 The womb chair

Analysis of the methods of metaphorical furniture design reveals that human cognition and emotions interact with each other, but emotions are abstract. Thus, the emotions are turned into metaphors in the shape of the products on which people can project their feelings and beliefs. As emotions differ, the requirements for metaphorical design differ. The demand decides the supply, and then, what are the requirements in different contexts?

\section{EMOTIONAL CHARACTERISTICS OF FURNITURE IN THE MARKET}

How can a design invoke emotions, and what emotions can be invoked? Most of the time, the user cannot express the characteristics they need accurately by language, and in these cases, the designer must capture the users' needs, and convert these needs into design elements. The real value of products is to satisfy the needs of the users and give them a sense of joy. As Donald Norman states, "a product must be appealing, joyous and interesting, as well as effective and understandable". This shows the importance of design to people's emotional needs ${ }^{[5]}$. Designers must have an in-depth understanding of the using habits of users in different occasions.

Furniture has different meanings in social situations. Human-centered knowledge is built based on design activities. Furniture can be divided into three categories as per the occasion they serve: personal place, workplace, public place. Home is a place full of love, and the style of furniture at home differs as per the users' preferences. With love, a house turns into a home where people find a balance between work and life. There are furniture items that are comforting and show good tastes - a inspiring book shelf or a relaxing chair, simple, peaceful but comforting. Companies often choose quality furniture pieces that will enhance the work experience of the employees. Furniture at the workplace matches the style of the work, independent, secure and convenient. Meanwhile, the furniture at the workplace has high standards for quality and comfort, so furniture pieces of ergonomic design are extensively used in the workplace, with enjoyable shapes and texture. The furniture of public places, or urban furniture, also serves to decorate the city and carry on cultural traditions. Urban furniture is a form of urban art that reflects the geographic conditions and local culture of the city.

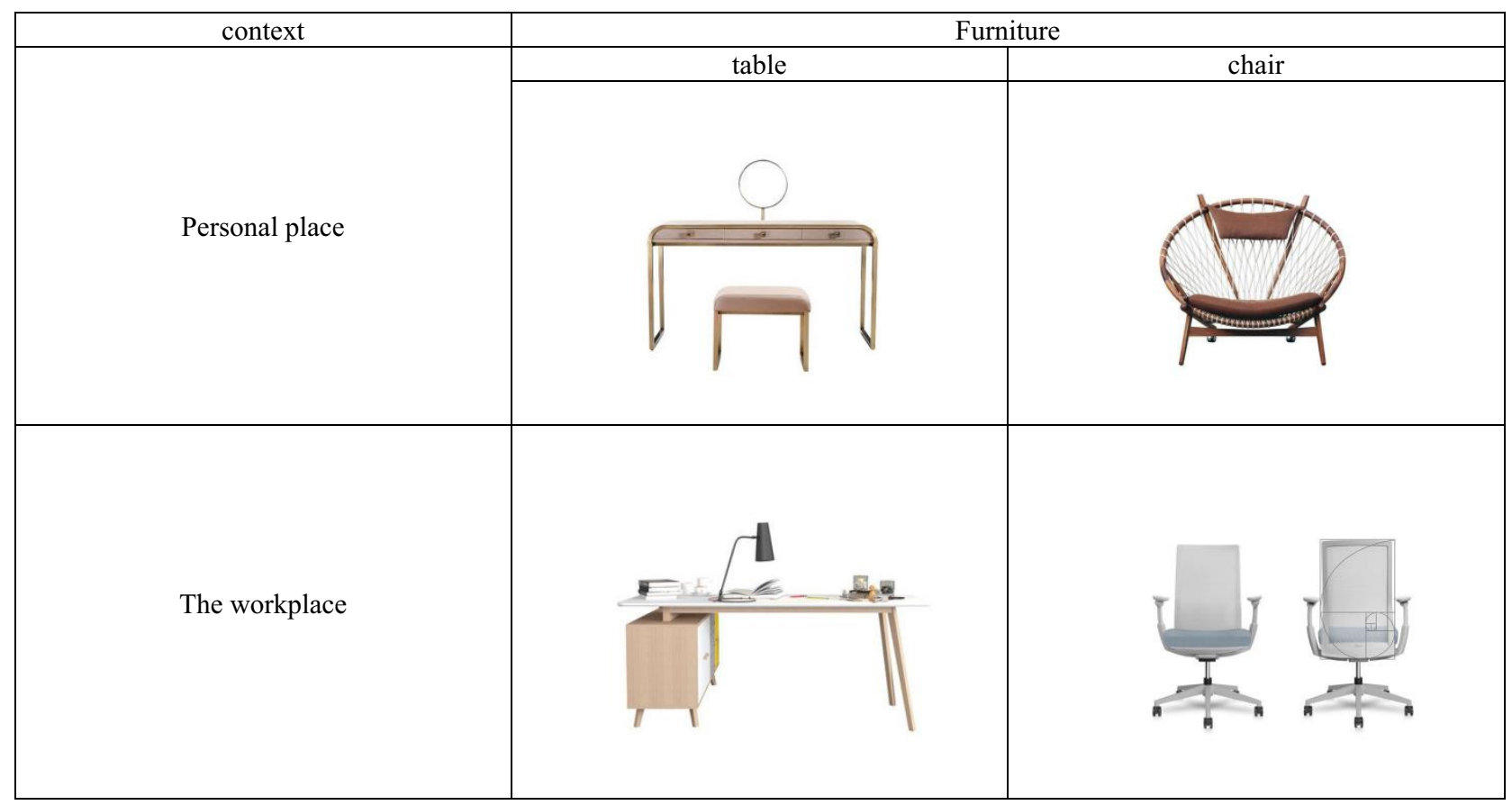




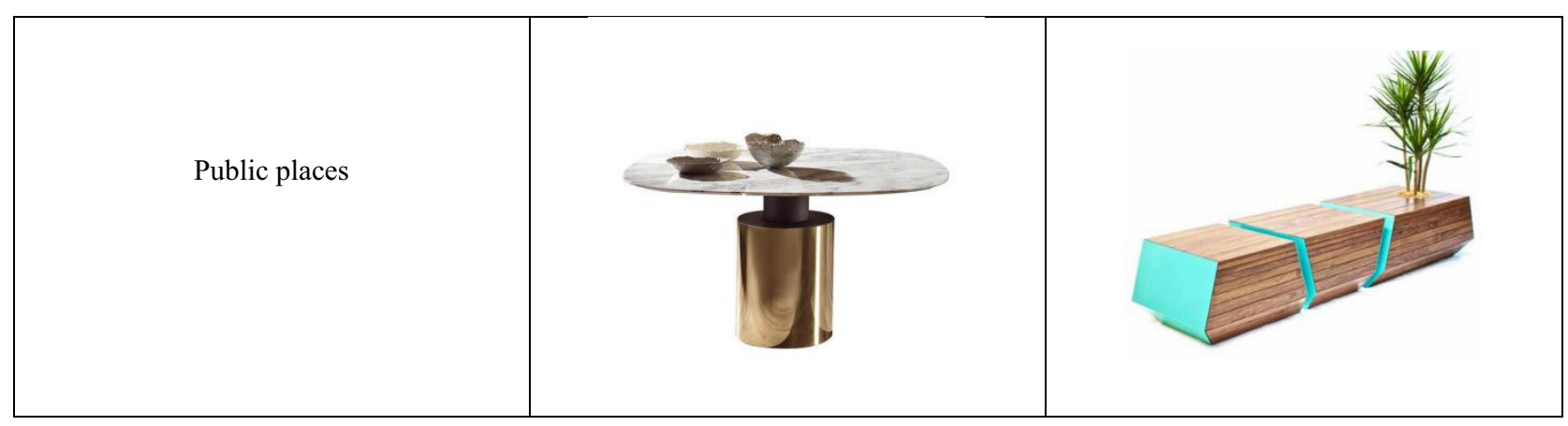

Fig. 5 Semantic analysis of furniture

\section{The Case of Parametric Technique in FURNITURE METAPHORICAL DESIGN}

Parametric technology refers to a computer-aided automatic manufacturing technology with given parameters and variables. The parametric technology can help designers produce highly innovative metaphorical designs that have distinct features and meet the users' specific needs, and thereby provide customized service. Grasshopper is uesd as parameterized editing software.

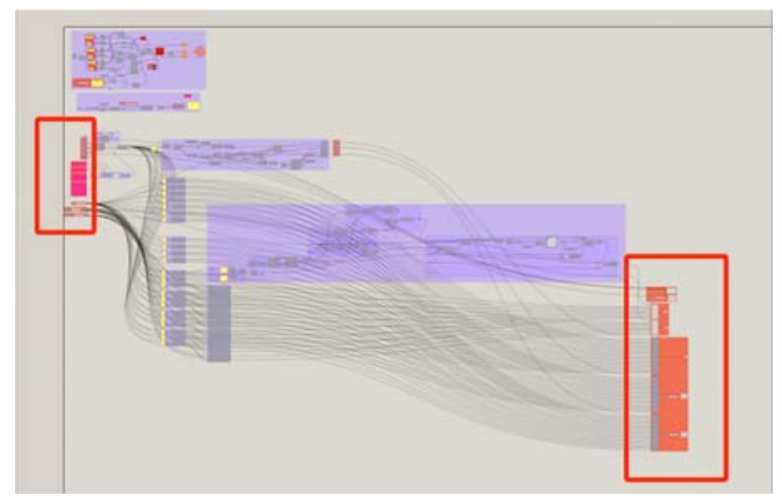

Figure 6. Grasshopper visualization algorithm

\subsection{Physical drawings and analysis}

When overwhelmed by pressure at work, people will try to strike a balance between work and life. One way to strike a balance is to discover beauty, at home. Staying at home has become a prevailing way of consumption among the generations born after 2000, so to improve the experience at home is critical in furniture design. Under the coronavirus pandemic, people are forced to stay at home, which gives rise to new scenes like "tours on the balcony", "tours at home", "artificial waterfalls", etc. on social media. People created contextual beauty at home dilute the disappointment of inability to go out. When enjoying life, we also need to protect the environment. The havoc that diseases and natural disasters have caused to the world reminds us to consider the impact of products on the environment during design activities, and tells us that design should be people-oriented. Many brands have set a good example in ecological protection. They use recycled materials or eco-friendly materials to create new composites, turn them into durable materials so that many recycled materials can be reused.

Firstly, the source of metaphor is collected and selected. Secondly, whether the semantic communication of metaphors is reasonable in context is analyzed, and whether the technology is feasible is considered to realize holistic design. When we grow up, we enjoy recalling the days hanging out with playmates in childhood. Some games like rubber band skipping, hide and seek, are cherished memories and are beneficial for health. Such games do not need extra resources, as long as we can find a place to play. During the play, the children benefit from their interactions, so these games are more down-to-earth than e-games. The author's design aims to bring the users back to their childhood days and draws inspiration from the game of throwing sand bags (Fig. 7). After a long day in the office, the sandbag chair will bring joy back to you, so it is named "Enjoy the Me Time" Sandbag chair. 


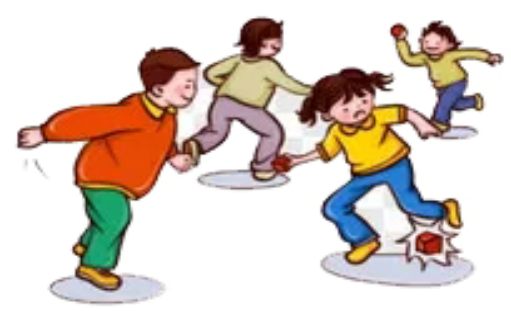

Fig. 7 From story to innovation

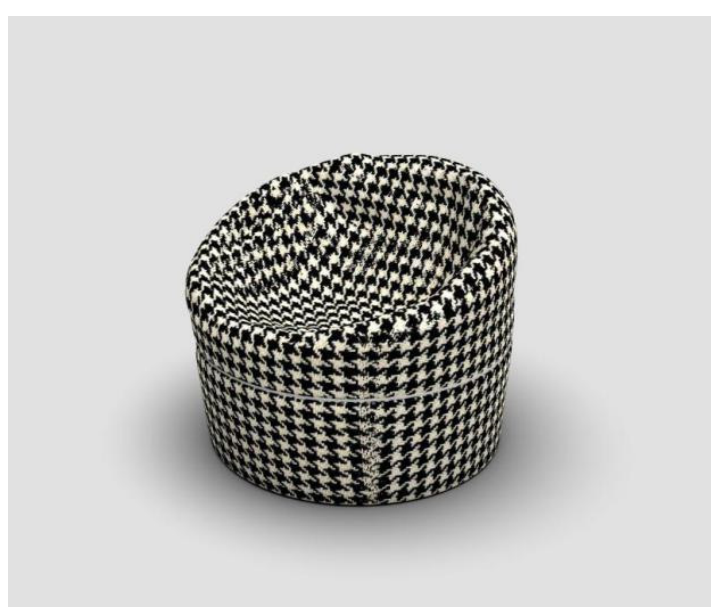

Fig. 8 "Enjoy the Me Time" Sandbag chair
The parametric technology was employed for design and manufacturing. The raw materials must be carefully selected in the design of furniture. To ensure safety and protect the environment, furniture designers now prefer eco-friendly materials or new composites. When the pieces are used the parts of the furniture can be disassembled, decomposed and reused, which conform to the idea of green design. In the author's design, the author selects eco-friendly raw material of the buckwheat skin. The buckwheat skin is the shell of buckwheat, which is elastic, resistant to metamorphism, rhombus-shaped, firm but not hard, and resistant from being broken. There are spaces between the shells to form air passages. It is often used as pillow fillings, can be removed or washed, and it is economical, and clean. The outer material is cloth, and a zipper is used to open or close the sandbag chair.

In the design stage, the design software Rhinoceros was used to adjust the parameters and the framework. He "Enjoy Me Time" sandbag chair was modelled based on the parametric technology in terms of the basic concepts, ergonomic factors, functions and structures of the design.

From story to innovation (Fig. 6), the design conveys the author's feelings about childhood to the user. Because of its light weight, this sandbag chair serves both as a chair to sit on and a toy for the family members to play with.

\section{Conclusion}

The appearance and texture are both expressions of a product, and every line serves to give expression to its features and functions. When buying a product, the customer is not just buying a physical object, but also something intangible, a design that fulfills their mental or spiritual needs, a design that meets their needs to show their identify, to follow the vogue, or to recall the old days. Emotion is an important concept in semantics, and metaphorical design is a transfer of emotions, which plays an important role in semantic communication ${ }^{[8]}$. The use of metaphor means, may produce some shape, in the design and manufacture of shape to use parametric design, parameterization at least at this stage, not to solve the problem, but to provide new and many options.

\section{References}

1. Chen Shenren, Ma Haibo. Design Morphological Semantics -- Artistic Morphological Semantics [M]. Chemical Industry Press, 2005-1.

2. Shu Dingfang. Research on Metaphor [M]. Shanghai: Shanghai Foreign Language Education Press,2000.

3. Gao Zhenwei, GAO Yuanhua. Semantic Design of Product Form [M]. Hefei Industrial Press,

4. Klaus Krippendorf. Design: Semantic Transformation [M]. China Building Industry Press,

5. Donald A. Norman. Emotional design [M]. Electronic Industry Press, 2005.

6. [By] George Lakoff / [by] Mark Johnson. The metaphor by which we live [M]. Zhejiang University Press, 2015.4.

7. Wu Feng. Art Symbol Aesthetics [M]. Beijing Broadcasting Institute Press, 2002.

8. Susan Langer. Emotion and Form [M]. Nanjing Press, 2006-1.

9. Dai Xinwei. Study on the Application of Grasshopper-based Parametric Design in Product Design [J].]; and Design ,2016(11):122-123. 